\title{
A non-intrusive approach of goal-oriented error estimation for evolution problems solved by the finite element method
}

\author{
Ludovic Chamoin* — Pierre Ladevèze*,** \\ * LMT-Cachan (ENS Cachan/CNRS/Paris 6/PRES Univ. Sud-Paris) \\ 61 av. du Président Wilson, F-94230 Cachan \\ \{chamoin,ladeveze\}@lmt.ens-cachan.fr \\ ** EADS Foundation Chair “Advanced Computational Structural Mechanics”
}

\begin{abstract}
In this article, we set up a non-intrusive procedure that yields for strict and highquality error bounds of quantities of interest in linear viscoelasticity problems solved by means of the Finite Element Method. The non-intrusive feature is achieved by introducing, via a partition of unity, enrichment functions in the solution of the adjoint problem (handbook techniques). The resulting goal-oriented error estimation method is thus easy to implement in a FE code and enables to consider trully pointwise quantities of interest.

RÉSUMÉ. Nous présentons dans cet article une procédure permettant d'obtenir de façon non intrusive des bornes à la fois garanties et pertinentes de l'erreur sur des quantités locales pour les problèmes de viscoélasticité linéaire résolus par la méthode des élements finis. Le caractère non intrusif est apporté par l'introduction de fonctions d'enrichissement, à l'aide d'une partition de l'unité, lors de la résolution du problème adjoint (techniques "handbook»). La méthode d'estimation d'erreur locale qui en découle est alors simple à implémenter dans un code de calcul et permet de considérer des quantités d'intérêt véritablement ponctuelles.

KEYWORDS: verification, Local error, strict bounds, non-intrusive methods, handbook techniques, pointwise quantities of interest.

MOTS-CLÉS : vérification, erreur locale, bornes garanties, méthodes non intrusives, techniques handbook, quantités ponctuelles.
\end{abstract}

DOI:10.3166/REMN.17.981-992 @ 2008 Lavoisier, Paris

REMN - 17/2008. Giens 2007, pages 981 to 992 


\section{Introduction}

In the widespread numerical simulations carried out nowadays, a major concern remains the control of the quality of the numerical solutions obtained through approximate methods. Since the 70s, effective tools have emerged to assess global discretization error (Babuška et al., 2001; Ladevèze et al., 2004). Today, research intensely focuses on goal-oriented error estimation, i.e. assessment of the error on specific local quantities which are relevant for design purposes. The most accomplished works deal with linear static problems and give effective local error bounds (Paraschivoiu et al., 1997; Prudhomme et al., 1999; Pares et al., 2006). However, very few works on the subject are dedicated to evolution and non-linear problems; furthermore these usually lead to bounds which lack reliability because they are not guaranteed and/or not sharp, which is a serious drawback for robust design.

In the framework of linear viscoelasticity problems described through internal variables and solved by means of the Finite Element Method (FEM), we introduced in (Chamoin et al., 2007) a method that yields for strict and effective error bounds on local quantities. This method, which is an expansion of the basic ones given in (Ladevèze, 2006; Ladevèze, 2008), leans on classical extraction techniques (leading to the solution of an adjoint problem), the concept of dissipation error and convexity properties. It takes history effects into account and may lead to very sharp error bounds provided that the adjoint problem is solved securately. A simple but intrusive way of reaching such an accurate solution consisted in a local refinement of the time/space mesh being used for the adjoint problem (Chamoin et al., 2007).

In this paper, we go a step further by setting up a non-intrusive procedure to solve the adjoint problem precisely, in the sense that we keep unchanged the discretization parameters (mesh, operators) defined for the reference (or primal) problem (Chamoin et al., 2008). We use for that handbook techniques (Strouboulis et al., 2000) which consist in introducing enrichment functions via the Partition of Unity Method (PUM) when solving the adjoint problem with the FEM. These functions correspond to locally (quasi-)exact solutions of the adjoint problem; they are computed analytically or numerically in a (semi-)infinite domain. As a result, we get high-quality error bounds at low cost without any remeshing. Furthermore, the method enables to consider trully pointwise quantities of interest in space and time by using as enrichment functions the well-known and possibly infinite energy Green functions.

\section{Reference problem and dissipation error}

\subsection{The reference problem}

We consider the structure $\Omega$, with boundary $\partial \Omega$, given in Figure 1. It is subject over the time interval $[0, T]$ to prescribed time-dependent mechanical sollicitations $\left(\underline{U}_{d}, \underline{f}_{d}, \underline{F}_{d}\right)$. We assume that this loading is zero at $t=0$ and that its evolution with time is piecewise linear. 


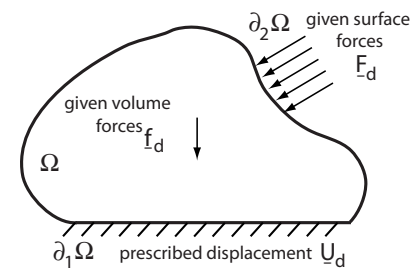

(a)

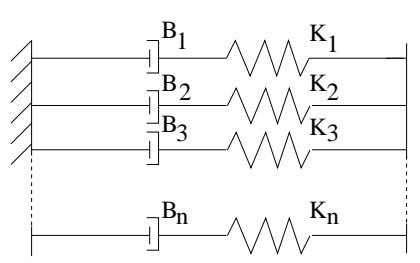

(b)

Figure 1. Structure and its environment (a), and rheological model used (b)

We choose a material with a linear viscoelastic behavior defined by the generalized Maxwell model. The associated rheological model (Figure 1) is constituted of an assembly of $n$ spring/damper sets. Such a material model can be easily described by means of the generalized internal variables ( $n$-vectors):

$$
s=\left[\begin{array}{c}
\sigma_{1} \\
\cdot \\
\sigma_{n}
\end{array}\right] \quad e^{e}=\left[\begin{array}{c}
\epsilon_{1}^{e} \\
\cdot \\
\epsilon_{n}^{e}
\end{array}\right] \quad e^{p}=\left[\begin{array}{c}
\epsilon_{1}^{p} \\
\cdot \\
\epsilon_{n}^{p}
\end{array}\right] \quad e=e^{e}+e^{p}
$$

where $\epsilon_{i}^{e}$ and $\epsilon_{i}^{p}$ are respectively the elastic and anelastic part of the total strain $\epsilon_{i}=$ $\epsilon(\underline{u})$ in set $i$, whereas $\sigma_{i}$ are the dual variables related to the Cauchy stress tensor $\sigma$ by $\sum_{i=1}^{n} \sigma_{i}=\sigma$. With these notations, the intrinsic dissipation $d$ of the model reads:

$$
d=\sum_{i=1}^{n} \operatorname{Tr}\left[\sigma_{i} \dot{\epsilon}_{i}^{p}\right]=s \cdot \dot{e}^{p} .
$$

Under the assumptions of quasi-static, isothermal and small perturbations state, the reference problem consists in finding a solution $(e, s)$ that verifies:

- the compatibility equations: $e$ is kinematically admissible ( $e \mathrm{KA}$ );

- the equilibrium equations: $s$ is statically admissible $(s \mathrm{SA})$;

- the initial conditions;

- the constitutive relations which are split into two parts:

$$
\begin{gathered}
e^{e}=\boldsymbol{\Lambda}(s) ; \quad \sum_{i=1}^{n} \sigma_{i}=\sigma \quad \text { (state equations); } \\
\dot{e}^{p}=\boldsymbol{B}(s) \quad \text { (evolution laws). }
\end{gathered}
$$

The exact solution of the reference problem, denoted by $\left(e_{e x}, s_{e x}\right)$, can not usually be reached. We thus compute an approximate solution, denoted by $\left(e_{h}, s_{h}\right)$, of the problem using the FEM associated to a backward-Euler scheme. For that, we divide the time interval $[0, T]$ into $N$ time steps $\left[t_{k}, t_{k+1}\right](k=0, \ldots, N-1)$ and we interpolate over $[0, T]$ the approximate solution $\left(e_{h, k}, s_{h, k}\right)$ obtained at each time point $t_{k}$. Therefore, we define the discretization error $\underline{u}_{e x}-\underline{u}_{h}$. 


\subsection{Computation of the dissipation error}

The concept of dissipation error requires the possession of a solution $(\hat{e}, \hat{s})$, called an admissible solution, that should verify all the equations of the reference problem except the evolution laws [2]. Such a solution can be built from the FE solution $\left(e_{h}, s_{h}\right)$ computed previously, using techniques developed at LMT-Cachan for many years (Ladevèze et al., 2004). Dissipation error $E_{\text {diss }}$ is thus a global measurement of the non-verification, for a given admissible solution $\left(\hat{e}_{h}, \hat{s}_{h}\right)$, of the evolution laws. It reads:

$$
E_{d i s s}^{2}\left(\hat{e}_{h}, \hat{s}_{h}\right)=\frac{1}{2} \int_{0}^{T} \int_{\Omega} a(t)\left(\dot{\hat{e}}_{h}^{p}-\boldsymbol{B}\left(\hat{s}_{h}\right)\right) \cdot \boldsymbol{B}^{-1}\left(\dot{\hat{e}}_{h}^{p}-\boldsymbol{B}\left(\hat{s}_{h}\right)\right) \mathrm{d} \Omega \mathrm{d} t .
$$

The time function $a(t)$, which is positive over $[0, T]$, has been added to the original definition of the dissipation error given in (Ladevèze et al., 2004); this enables to get a weighted dissipation error that takes the history effects encountered in evolution problems into account (Chamoin et al., 2007). A first property of the dissipation error is that it represents a global discretization error estimator which accounts for all sources of error (time and space discretizations in our case). Another property, which is the true engine to get strict local error bounds, is the link between $E_{d i s s}\left(\hat{e}_{h}, \hat{s}_{h}\right)$ and the exact solution $\left(e_{e x}, s_{e x}\right)$; it is on the form $G\left(s_{e x}-\hat{s}_{h}\right)=E_{\text {diss }}^{2}\left(\hat{e}_{h}, \hat{s}_{h}\right)$ where $G$ is a given functional based on free energy and pseudo-potentials of dissipation.

\section{The goal-oriented error estimation method}

The quantities of interest we deal with may be local in space and time and are dedicated to viscoelasticity problems. We only consider here quantities which depend linearly on components of $s$ or $e$, such as a component of the stress $\sigma$, of the displacement $\underline{u}$, of an internal variable $\epsilon_{i}^{p}$ or of its rate $\dot{\epsilon}_{i}^{p}$ at time $t \in[0, T]$. However, the case of nonlinear quantities of interest may not be an issue provided that they are local.

In the framework of the extraction techniques we use, the first step consists in writing the considered quantity of interest $I$ in a global form:

$$
I=\int_{0}^{T} \int_{\Omega} \sum_{i=1}^{n} \operatorname{Tr}\left[\sigma_{i} \dot{\tilde{\epsilon}}_{\Sigma i}\right] \mathrm{d} \Omega \mathrm{d} t=\left\langle\left\langle s, \dot{\tilde{e}}_{\Sigma}\right\rangle\right\rangle=-\left\langle\left\langle\dot{e}, \tilde{s}_{\Sigma}\right\rangle\right\rangle .
$$

The $n$-vectors $\dot{\tilde{e}}_{\Sigma}$ and $\tilde{s}_{\Sigma}$, known analytically, represent the extraction function. Following the procedure described in (Becker et al., 2001), we then define a new problem, called adjoint problem, which is reverse in time but remains similar to the reference problem except that the loading now consists in the prestress $\tilde{\sigma}_{\Sigma}=\sum_{i=1}^{n} \tilde{\sigma}_{\Sigma i}$. The adjoint problem thus boils down to finding a solution $(\tilde{e}, \tilde{s})$ that verifies:

- the compatibility equations: $\tilde{e} \mathrm{KA}-0$;

- the equilibrium equations: $\tilde{s}-\tilde{s}_{\Sigma} \mathrm{SA}-0$; 
- the final conditions;

- the constitutive relations (state equations, evolution laws $\dot{\tilde{e}}^{p}=-\boldsymbol{B}(\tilde{s})$ ).

In the same way as for the reference problem, we compute a FE solution $\left(\tilde{e}_{h}, \tilde{s}_{h}\right)$ and an admissible solution $\left(\hat{\tilde{e}}_{h}, \hat{\tilde{s}}_{h}\right)$ for the adjoint problem. Note that the time/space mesh used to solve the adjoint problem can be chosen independently from the one defined for the reference problem. The following result thus yields (technical details can be found in (Chamoin et al., 2007)):

$$
\left|I_{e x}-I_{h}-I_{h h}\right| \leq 2\left[\frac{1}{2} E_{\text {diss }}^{2}\left(\hat{e}_{h}, \hat{s}_{h}\right)+F_{0}\left(\Delta_{h}\right)\right]^{\frac{1}{2}} \cdot\left[F_{2}\left(\tilde{x}_{h}\right)\right]^{\frac{1}{2}}
$$

where $I_{e x}$ (resp. $I_{h}$ ) is the unknown exact value (resp. FE value) of the quantity of interest $I, I_{h h}$ is a correction term computed from the approximate solutions of both reference and adjoint problems, $F_{0}$ and $F_{2}$ are some functions known analytically, $\Delta_{h}$ is a computable term that is not explicited here, and $\tilde{x}_{h}=-\boldsymbol{B}\left(\hat{\tilde{s}}_{h}\right)-\dot{\tilde{\tilde{e}}}_{h}^{p}$.

As a result, we obtain from [5] some strict bounds $\xi_{\text {inf }}$ and $\xi_{\text {sup }}$ of $I_{\text {ex }}$, equal to:

$$
I_{h}+I_{h h} \pm 2\left[\frac{1}{2} E_{\text {diss }}^{2}\left(\hat{e}_{h}, \hat{s}_{h}\right)+F_{0}\left(\Delta_{h}\right)\right]^{\frac{1}{2}} \cdot\left[F_{2}\left(\tilde{x}_{h}\right)\right]^{\frac{1}{2}} .
$$

REMARK. - The quantity $I_{h}+I_{h h}$ can be viewed as a new approximation of $I_{e x}$.

Bounds $\xi_{\text {inf }}$ and $\xi_{\text {sup }}$ are sharp provided that term $F_{2}\left(\tilde{x}_{h}\right)$ is small enough, i.e. when the adjoint problem is solved correctly. This can be reached by refining locally the time/space mesh used to solve the adjoint problem. However, this intrusive technique may lead to large modifications in a FE code, which is a drawback. Consequently, we rather set up a non-intrusive technique that is explained in Section 4.

\section{Non-intrusive approach for the solution of the adjoint problem}

\subsection{General framework}

Usually, the loading of the adjoint problem induces solutions presenting singularities or high gradients in some localized zones of the domain $[0, T] \times \Omega$. It is thus difficult to represent these solutions properly with a classical FE discretization. We propose here a procedure based on the handbook techniques developed in (Strouboulis et al., 2000). It consists in introducing enrichment functions, via the Partition of Unity Method (PUM), in the set of basis functions describing the approximate displacement field. These functions are singular solutions $\left(\tilde{e}^{\text {hand }}, \tilde{s}^{\text {hand }}\right)$ of the adjoint problem loading over an infinite (or semi-infinite) domain; they are usually computed analytically in time and numerically in space and constitute a library of pre-calculated solutions. They only depend on the quantity of interest considered, and on the characteristic coefficients of the material. However, these dependences are quite easy to account for due to the analytical definition of the functions (time evolution at least) and the linearity of the material behavior. 
Therefore, we now search a displacement field for the adjoint problem under the form:

$$
\underline{\tilde{u}}=\sum_{j=1}^{n n o^{P U M}} \psi_{j} \underline{\tilde{u}}^{\text {hand }}+\underline{\tilde{u}}^{r}
$$

where $\psi_{j}$ is the classical FE shape function associated to node $j, n n o^{P U M}$ is the total number of nodes enriched by the PUM and $\underline{\tilde{u}}^{r}$ is a displacement field to be calculated. Note that the degrees of freedom associated to the PUM are known i.e. the enrichment is entirely determined; only the field $\underline{\tilde{u}}^{r}$ is unknown. The total solution $(\tilde{e}, \tilde{s})$ then reads:

$$
(\tilde{e}, \tilde{s})=\left(\tilde{e}_{P U M}^{h a n d}, \tilde{s}_{P U M}^{\text {hand }}\right)+\left(\tilde{e}^{r}, \tilde{s}^{r}\right) .
$$

It is composed of two terms:

- an enrichment term $\left(\tilde{e}_{P U M}^{\text {hand }}, \tilde{s}_{P U M}^{\text {hand }}\right)$ which locally equilibrates the loading of the adjoint problem. However, it does not verify all the boundary conditions on $\partial \Omega$;

- a FE term $\left(\tilde{e}_{h}^{r}, \tilde{s}_{h}^{r}\right)$ which can be seen as a residual solution and that enables to verify all the boundary conditions on $\partial \Omega$.

We denote by $\Omega^{P U M}$ the part of the domain $\Omega$ involved in the enrichment by the PUM. It is split into a part $\Omega_{1}^{P U M}$, such that $\sum_{j=1}^{n n o^{P U M}} \psi_{j}(M)=1 \quad \forall M \in \Omega_{1}^{P U M}$, that contains the area of the structure over which the loading of the adjoint problem is applied, and a part $\Omega_{2}^{P U M}$ that is the complementary part of $\Omega_{1}^{P U M}$ in $\Omega^{P U M}$ (Figure 2).

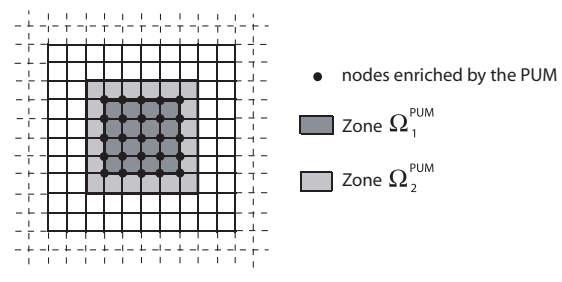

Figure 2. Definition of the zones introduced with the PUM

The new problem we thus have to solve consists in finding the residual solution $\left(\tilde{e}^{r}, \tilde{s}^{r}\right)$. It retains the same structure as the original adjoint problem except that the loading is changed, i.e. the equilibrium equation now reads:

$$
\begin{aligned}
\int_{\Omega} \operatorname{Tr}\left[\tilde{\sigma}^{r} \epsilon\left(\underline{u}^{*}\right)\right] \mathrm{d} \Omega= & -\int_{\partial \Omega_{1}^{P U M}} \tilde{\sigma}^{h a n d} \underline{n}_{12} \cdot \underline{u}^{*} \mathrm{~d} \Omega \\
& -\int_{\Omega_{2}^{P U M}} \operatorname{Tr}\left[\tilde{\sigma}_{P U M}^{h a n d} \epsilon\left(\underline{u}^{*}\right)\right] \mathrm{d} \Omega \quad \forall t \in[0, T] \quad \forall \underline{u}^{*} \in \mathcal{U}_{0}
\end{aligned}
$$

where $\underline{n}_{12}$ is the outgoing unit normal vector on $\partial \Omega_{1}^{P U M}$. Due to the relative smoothness of the residual solution $\left(\tilde{e}^{r}, \tilde{s}^{r}\right)$, we can compute an accurate approximate solution $\left(\tilde{e}_{h}^{r}, \tilde{s}_{h}^{r}\right)$ using the FEM with the same time/space discretization as the one used for 
the reference problem. The method is called non-intrusive in this sense: we reuse the operators (factorized stiffness matrix, ... ) of the reference problem and only the force vector has to be changed. The user intervention is necessary only to define the quantity of interest; it is not required to produce the force vector which depends on the enrichment function and the enrichment zone. Practically, the adjoint problem is solved in the same time as the reference problem. Eventually, we get an approximate solution $\left(\tilde{e}_{h}, \tilde{s}_{h}\right)$ of the adjoint problem, such that $\left(\tilde{e}_{h}, \tilde{s}_{h}\right)=\left(\tilde{e}_{P U M}^{\text {hand }}, \tilde{s}_{P U M}^{\text {hand }}\right)+\left(\tilde{e}_{h}^{r}, \tilde{s}_{h}^{r}\right)$. After computing an admissible residual solution $\left(\hat{\tilde{e}}_{h}^{r}, \hat{\tilde{s}}_{h}^{r}\right)$, the bounding result [5] holds with:

$$
\tilde{x}_{h}=-\boldsymbol{B}\left(\hat{\tilde{s}}_{h}\right)-\dot{\tilde{\tilde{e}}}_{h}^{p}=-\boldsymbol{B}\left(\hat{\tilde{s}}_{h}^{r}\right)-\dot{\tilde{\tilde{e}}}_{h}^{r, p}
$$

due to the fact that the evolution laws are verified by the handbook solutions. As regards term $I_{h h}$ involved in [5], it is calculated using overintegration.

\subsection{Case of pointwise quantities of interest}

The extension of the non-intrusive method presented above to pointwise in space quantities of interest is straightforward. Indeed, the loading of the adjoint problem (force, prestress...) being also pointwise in space in that case, the associated handbook functions correspond to the well-known Green functions. One can introduce such functions, even though they are infinite-energy, into the approximate solution of the adjoint problem as they do not appear in the expression of the dissipation error related to the adjoint problem. The Green functions are here calculated analytically in space and time, using a method based on strain nuclei (Love, 1944) which leads to the calculation of Galerkin vectors that are solutions of a simple biharmonic equation. An example of such a Green function over an infinite domain is given in Figure 3. When dealing with a semi-infinite domain, we use the image method that consists in taking contributions of other singular sollicitations into account (Sneddon et al., 1964).

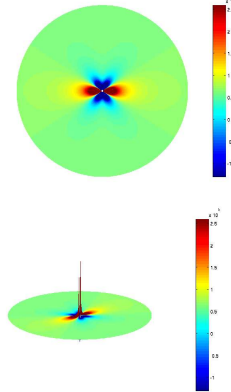

(a)
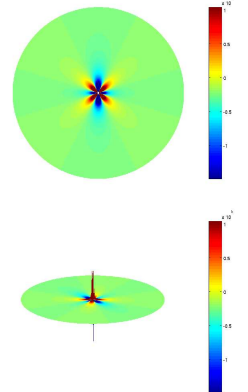

(b)
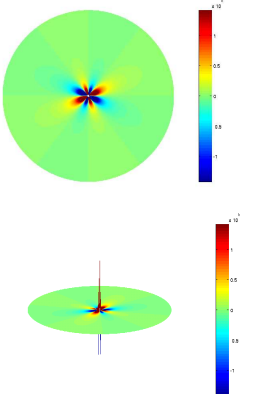

(c)

Figure 3. Spatial distribution of the stress field corresponding to a pointwise prestress loading over a $2 D$ infinite domain: $\tilde{\sigma}_{x x}^{\text {hand }}(a), \tilde{\sigma}_{y y}^{\text {hand }}(b), \tilde{\sigma}_{x y}^{\text {hand }}(c)$ 
Even though the FE value $I_{h}$ of a pointwise quantity at some point $\mathrm{P}$ within $\Omega$ is not always defined (due to possible discontinuities of the derivatives across element boundaries), the bounding method can be applied. Indeed, [5] can be rewritten as:

$$
\left|I_{e x}-\hat{I}_{h}-\hat{I}_{h h}\right| \leq 2\left[\frac{1}{2} E_{d i s s}^{2}\left(\hat{e}_{h}, \hat{s}_{h}\right)+F_{0}\left(\Delta_{h}\right)\right]^{\frac{1}{2}} \cdot\left[F_{2}\left(\tilde{x}_{h}\right)\right]^{\frac{1}{2}}
$$

where $\hat{I}_{h}$ and $\hat{I}_{h h}$ are some quantities defined at any regular point $\mathrm{P}$ using the admissible solution $\left(\hat{e}_{h}, \hat{s}_{h}\right)$. Then, one can use the bounds of $I_{e x}$ given by [7]. As in Section 4.1, one has $\tilde{x}_{h}=-\boldsymbol{B}\left(\hat{\tilde{s}}_{h}^{r}\right)-\dot{\hat{\tilde{e}}}_{h}^{r, p}$ and the calculation of the dissipation error for the adjoint problem requires the smooth residual solution $\left(\hat{\tilde{e}}_{h}^{r}, \hat{\tilde{s}}_{h}^{r}\right)$ alone.

\section{Numerical results}

In this section, we apply the non-intrusive error estimation method to a $2 \mathrm{D}$ problem. We consider a L-shaped structure clamped at its base and subject to a prescribed displacement $\underline{U}_{d}(t)$ along its upper right edge (Figure 4).

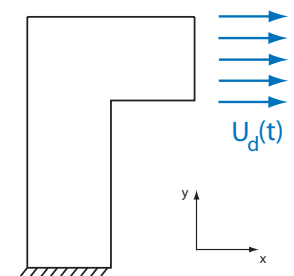

(a)

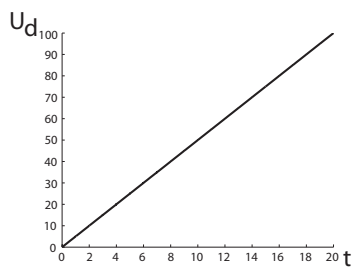

(b)

Figure 4. The structure being considered (a) and its loading (b)

We assume plane stress state and take a Maxwell rheological model composed of three spring/damper sets. The FE solution is obtained by discretizing the structure spatially with 100 linear quadrangle elements and dividing the time interval $[0, T]$ ( $T=20 \mathrm{~s}$ ) into 20 time steps. Let us note that the calculation of $I_{e x}$, used as the reference value, is performed using a "quasi-exact" solution obtained by means of a very refined FE mesh ("overkill solution").

\subsection{A first example}

We consider the quantity of interest:

$$
I=\frac{1}{|\omega|} \int_{\omega} \dot{\epsilon}_{1_{y y} \mid T}^{p} \mathrm{~d} \omega
$$


where $\omega$ corresponds to one-quarter of an element of the FE mesh (Figure 5). The loading of the associated adjoint problem consists in a prestress $\tilde{\sigma}_{\Sigma}$ in $\omega$.

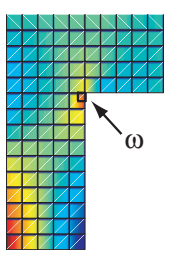

(a)
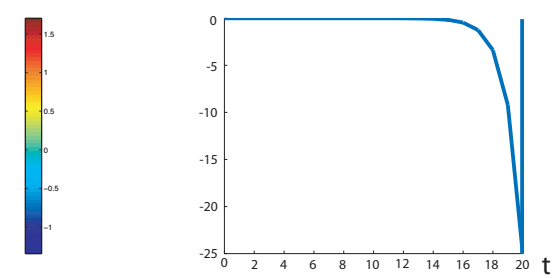

(b)

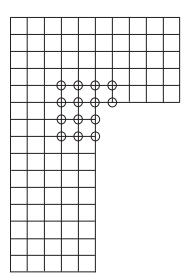

(c)

Figure 5. Map of Field $\dot{\epsilon}_{1_{y y}}^{p}$ at time $T(a)$, evolution in time of $\tilde{\sigma}_{\Sigma}(b)$, and nodes involved in the enrichment through the PUM (c)

We then introduce in the solution of the adjoint problem specific handbook functions calculated analytically in time and numerically in space. They represent the (quasi-)exact solution of the adjoint problem loading over a semi-infinite domain, taking the L-shape and the local traction-free conditions into account (Figure 6).

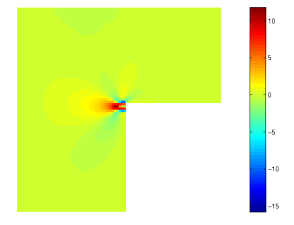

(a)

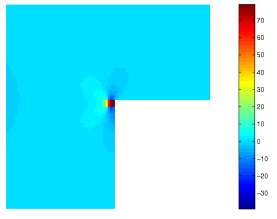

(b)

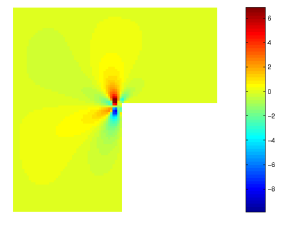

(c)

Figure 6. Map of $\tilde{\sigma}_{x x}^{\text {hand }}(a), \tilde{\sigma}_{y y}^{\text {hand }}(b)$, and $\tilde{\sigma}_{x y}^{\text {hand }}(c)$ at time $T$

The enrichment functions are introduced through the PUM at specific nodes of the mesh which are close to the zone of application of the adjoint problem loading (these nodes are circled in Figure 5).

We show in Figure 7 and Figure 8 the spatial distribution of Fields $\tilde{\sigma}_{P U M}^{\text {hand }}$ and $\tilde{\sigma}_{h}^{r}$ (such that $\tilde{\sigma}_{h}=\tilde{\sigma}_{P U M}^{\text {hand }}+\tilde{\sigma}_{h}^{r}$ ) at time $t=T$.

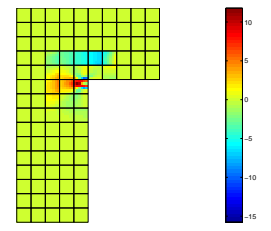

(a)

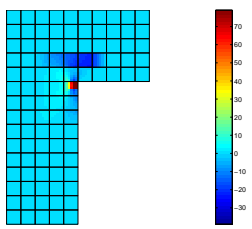

(b)

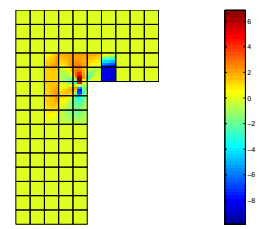

(c)

Figure 7. Map of $\tilde{\sigma}_{P U M_{x x}}^{\text {hand }}(a), \tilde{\sigma}_{P U M_{y y}}^{\text {hand }}(b)$, and $\tilde{\sigma}_{P U M_{x y}}^{\text {hand }}(c)$ at time $T$ 


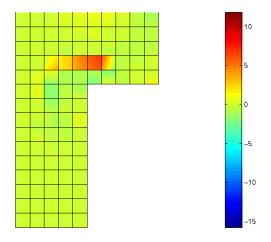

(a)

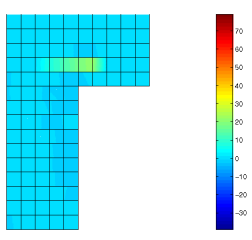

(b)

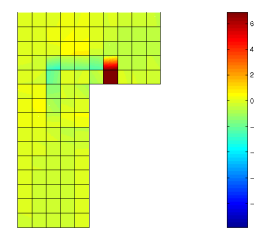

(c)

Figure 8. Map of $\tilde{\sigma}_{h_{x x}}^{r}(a), \tilde{\sigma}_{h_{y y}}^{r}(b)$, and $\tilde{\sigma}_{h_{x y}}^{r}(c)$ at time $T$

Eventually, we get the bounds:

$$
\bar{\xi}_{\text {inf }}=\frac{\xi_{\text {inf }}}{I_{\text {ex }}}=0.97 \quad \bar{\xi}_{\text {sup }}=\frac{\xi_{\text {sup }}}{I_{\text {ex }}}=1.02 .
$$

These results show that the non-intrusive method is very effective and enables to obtain accurate bounds of localized quantities through the enrichment of only a few nodes of the space mesh (due to St-Venant principle).

\subsection{Second example: error estimation on a pointwise quantity of interest}

We now consider the quantity of interest:

$$
I=\dot{\epsilon}_{1_{y y}}^{p}(\mathrm{P})_{T}
$$

where $\mathrm{P}$ is a point that lies within an element of the mesh (Figure 9).

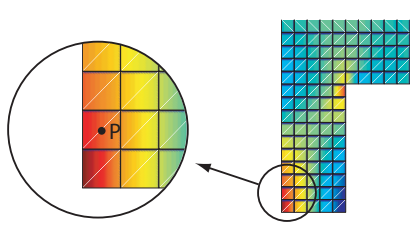

(a)

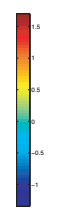

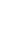

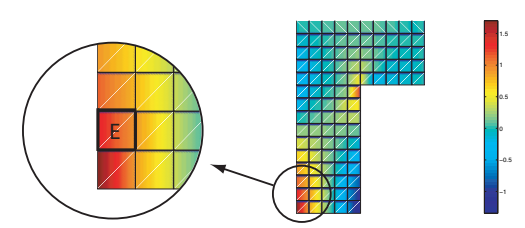

(b)

Figure 9. Definition of Point $P$ where the quantity of interest is defined (a), and definition of Zone $E(b)$

The loading of the adjoint problem consists in a pointwise prestress $\tilde{\sigma}_{\Sigma}(M, t)$ at Point $\mathrm{P}$ in the form $\tilde{\sigma}_{\Sigma}(t) \delta(\mathrm{P})$. The enrichment functions we use, taking traction-free boundary conditions into account, are similar to those given in Figure 3. 
We then get the following bounds:

$$
\bar{\xi}_{\text {inf }}=\frac{\xi_{\text {inf }}}{I_{\text {ex }}}=0,96 \quad \bar{\xi}_{\text {sup }}=\frac{\xi_{\text {sup }}}{I_{\text {ex }}}=1,04 .
$$

In addition, one can seek lower and upper bounds of $I_{e x}(\mathrm{P})$ for any point $\mathrm{P}$ within a specific local zone of interest $\mathrm{E} \subset \Omega$ (Figure 9 ). The procedure consists in sweeping Zone $\mathrm{E}$ and considering that the residual solution $\left(\tilde{e}_{h}^{r}, \tilde{s}_{h}^{r}\right)$ of the adjoint problem does not depend on the localization of P over $\mathrm{E}$ (practically, this is verified if zone $\Omega^{P U M}$ is sufficiently large). Thus only the handbook function has to be changed when sweeping over E, and the following result yields:

$$
\left|I_{e x}(\mathrm{P})-\hat{I}_{h}(\mathrm{P})-\hat{I}_{h h}(\mathrm{P})\right| \leq 2\left[\frac{1}{2} E_{\text {diss }}^{2}\left(\hat{e}_{h}, \hat{s}_{h}\right)+F_{0}\left(\Delta_{h}\right)\right]^{\frac{1}{2}} \cdot\left[F_{2}\left(\tilde{x}_{h}\right)\right]^{\frac{1}{2}} \quad \forall \mathrm{P} \in \mathrm{E}
$$

We thus get the following bounds for the extremum $I_{e x}^{\max , \mathrm{E}}$ of $I_{e x}$ over E:

$$
\bar{\xi}_{\text {inf }}^{\mathrm{E}}=\frac{\xi_{\text {inf }}^{\mathrm{E}}}{I_{e x}^{\text {max }, \mathrm{E}}}=0.95 \quad \bar{\xi}_{\text {sup }}^{\mathrm{E}}=\frac{\xi_{\text {sup }}^{\mathrm{E}}}{I_{e x}^{\text {max }, \mathrm{E}}}=1.05
$$

Therefore, we are able to obtain high-quality lower and upper bounds for the extremum of $I_{e x}$ (or $L^{\infty}$-norm of $I_{e x}$ ) over a given zone, which constitutes useful information for design purposes.

\section{Conclusion}

We presented in this paper a method that provides for strict and high-quality error bounds of local quantities in linear viscoelasticity problems. It is made non-intrusive due to the fact that by using handbook techniques, the adjoint problem is solved precisely while keeping unchanged the discretization parameters defined for the reference problem; only the loading has to be changed. As a result, the bounding process appears in a "black-box" manner for the analyst/designer. Furthermore, this technique enables one to easily tackle pointwise quantities by using Green's functions. Several numerical tests clearly illustrated the interest and efficiency of the proposed method.

In summary, this work demonstrates that reliable local error bounds can be obtained at reasonable cost for linear evolution problems, a fact which was not really accepted by the scientific community until now. It should also be mentioned that the goal-oriented error estimation method proposed here does not use the orthogonality properties of the FE solutions. Therefore, it could conceivably be applied to problems solved by approximate methods other than the FEM; it could moreover be applied to other linear parabolic problems.

\section{References}

Babuška I., Strouboulis T., The finite element method and its reliability, Oxford university press, 2001. 
Becker R., Rannacher R., “ An optimal control approach to shape a posteriori error estimation in finite element methods", A. Isereles (Ed.), Acta Numerica, Cambridge University Press, vol. 10, p. 1-120, 2001.

Chamoin L., Ladevèze P., " Bounds on history-dependent or independent local quantities in viscoelasticity problems solved by approximate methods", International Journal for $\mathrm{Nu}$ merical Methods in Engineering, vol. 71, n ${ }^{\circ}$ 12, p. 1387-1411, 2007.

Chamoin L., Ladevèze P., " A non-intrusive method to compute strict and efficient bounds of calculated outputs of interest in linear viscoelasticity problems", Computer Methods in Applied Mechanics and Engineering, vol. 197, $\mathrm{n}^{\circ}$ 9-12, p. 994-1014, 2008.

Ladevèze P., “ Upper error bounds on calculated outputs of interest for linear and nonlinear structural problems", Comptes Rendus Académie des Sciences - Mécanique, Paris, vol. 334, p. 399-407, 2006.

Ladevèze P., " Strict upper error bounds for computed outputs of interest in computational structural mechanics", Computational Mechanics, vol. 42, p. 271-286, 2008.

Ladevèze P., Pelle J., Mastering Calculations in Linear and Nonlinear Mechanics, Springer NY, 2004.

Love A., "A treatise on the mathematical theory of elasticity", Dover, New-York,p. 186-213, 1944.

Paraschivoiu M., Peraire J., Patera A., “ A posteriori finite element bounds for linear functional outputs of elliptic partial differential equations", Computer Methods in Applied Mechanics and Engineering, vol. 150, p. 289-312, 1997.

Pares N., Bonet J., Huerta A., Peraire J., " The computation of bounds for linear-functional outputs of weak solutions to the two-dimensional elasticity equations", Computer Methods in Applied Mechanics and Engineering, vol. 195, $\mathrm{n}^{\circ}$ 4-6, p. 406-429, 2006.

Prudhomme S., Oden J., “ On goal-oriented error estimation for elliptic problems: application to the control of pointwise errors", Computer Methods in Applied Mechanics and Engineering, vol. 176, p. 313-331, 1999.

Sneddon I., Hill R., Progress in solid mechanics, North Holland Publishing Company, Amsterdam, 1964.

Strouboulis T., Babuška I., Copps K., “ The design and analysis of the Generalized Finite Element Method", Computer Methods in Applied Mechanics and Engineering, vol. 181, p. 4369, 2000. 